\title{
Ultrasonic waves : a tool for gelation process measurements
}

\author{
J.-C. Bacri, J.-M. Courdille, J. Dumas and R. Rajaonarison \\ Laboratoire d'Ultrasons $\left({ }^{*}\right)$, Université Pierre-et-Marie-Curie, \\ Tour 13, 4 place Jussieu, 75230 Paris Cedex 05, France
}

(Reçu le 10 avril 1980, révisé le 30 mai, accepté le 13 juin 1980)

\begin{abstract}
Résumé. - Les ondes ultrasonores de haute fréquence $(\gtrsim 100 \mathrm{MHz})$ sont atténuées d'une manière très différente dans la phase sol ou la phase gel d'un milieu polymérique en cours de gélification, et fournissent donc un moyen très sensible et non destructif de suivre la transition sol-gel dans la phase gel : le point de gélification est précisément déterminé et la croissance de la fraction gel dans le milieu est suivie au cours de l'avancement de la réaction. Au prix de certaines hypothèses sur le coefficient de friction entre polymère et solvant, les mesures permettent de déterminer le coefficient critique $\beta$ qui gouverne la croissance de la fraction gel $S_{\infty}: \beta=0,5$.
\end{abstract}

Abstract. - The attenuation of high frequency ultrasonic waves ( $\gtrsim 100 \mathrm{MHz})$ is very different depending on the phase, sol or gel, of a polymeric melt in course of gelation, and its measurement is a sensitive and non destructive method for monitoring the sol-gel transition : the gelation point is accurately determined and the growing of the gel fraction is followed during the progress of the reaction.

With assumptions about the friction coefficient between polymer and solvent, our measurements give the critical coefficient $\beta$ of the gel fraction $S_{\infty}: \beta=0.5$.

The aim of this letter is to present some results obtained in the study of gelating system with ultrasonic waves.

The observation of the sol-gel transition is difficult for different reasons : the great softness of the gel fraction in the immediate vicinity of the threshold creates obstacles against applying macroscopic mechanical methods to this phase. The coherent inelastic light scattering technique which should be an ideal tool in this particular case is not usable because the fluctuation quantity near this transition is not the concentration but the connectivity, or the number of reticulating points, between the different polymer chains. In spite of certain possibilities given by incoherent scattering [1], some other methods must be found.

The measurement of the rotational diffusion constant of ferromagnetic particles imbedded in the solution provides a method for measuring the variation of macroscopic viscosity in the sol phase [2], and the elastic modulus behaviour of the gel fraction in the gel phase [3]. The same kind of information can also be obtained by more classical methods [4].

ique.
As we shall see, the ultrasonic wave attenuation is able to give some complementary insight into this phase, because as our experiments show elastic deformations have an excellent coupling with the property of connectivity.

1. Experimental results. - A classical pulsed ultrasonic system have been used : the solution of monomers, not yet reticulated, is inserted between two quartz rods acting as emission transducer and reception transducer respectively. Longitudinal elastic waves are used. Different spacers (from $100 \mu$ to $2 \mathrm{~mm}$ ) give a calibrated thickness to the sample. The ultrasonic attenuation is deduced from the measurement of the intensity of the ultrasonic signal transmitted through the sample. The choice of working frequencies $(110 \mathrm{MHz}$ and $790 \mathrm{MHz})$ has been made with two goals : to be well outside the monomer relaxation regions $(\simeq 10 \mathrm{MHz})$ and to insure a good sensitivity with the very small samples needed to have a reliable signal.

The sample is placed in a bath in which the temperature can be regulated between $-10^{\circ} \mathrm{C}$ and $+60^{\circ} \mathrm{C}$.

Two kinds of gelation have been examined :

- the chemical gelation of a $10 \%$ polyacrylamid gel is observed by recording the ultrasonic attenuation 
at $110 \mathrm{MHz}$ as a function of time, for two constant temperatures, $16^{\circ} \mathrm{C}$ and $20^{\circ} \mathrm{C}$. The sample is $2 \mathrm{~mm}$ thick (Figs. 1 and 2);

- the physical gelation of gelatin occurs when the temperature of the melt is lowered : the ultrasonic attenuation is recorded as a function of temperature. The measurements were made at $790 \mathrm{MHz}$ on $110 \mu$ thick samples for four different concentrations $(11 \%$, $7 \%, 5.3 \%, 2 \%$ ) (Fig. 3).

The methods of preparation of the solution of monomers are classical and have been already given elsewhere $[2,5]$.

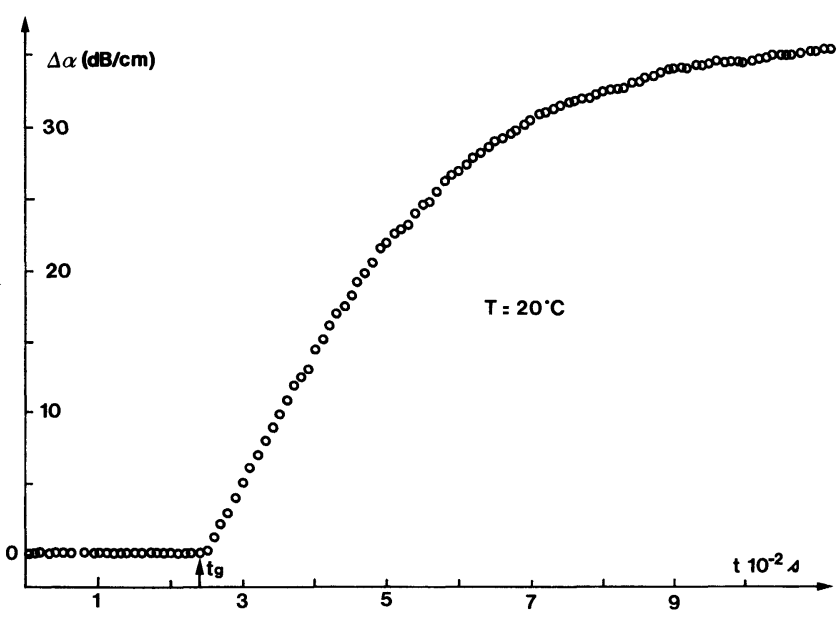

Fig. 1. - Ultrasonic attenuation variation during the gelation process in a polyacrylamid gel. $t_{\mathrm{g}}$ is the gelation threshold $\left(T=20^{\circ} \mathrm{C}\right.$, $v=110 \mathrm{MHz}$ ). At $t=0$ the attenuation is that of the monomer solution.

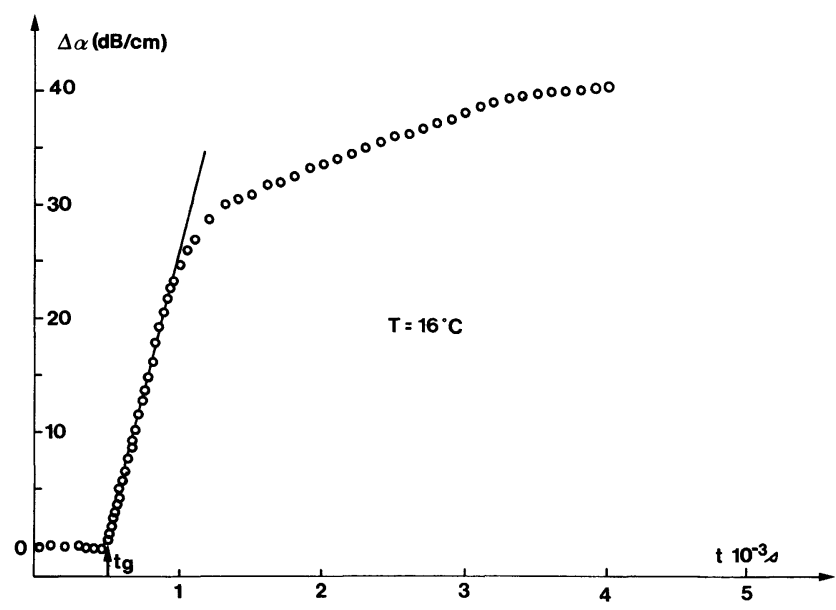

Fig. 2. - Ultrasonic attenuation variation during the gelation process in a polyacrylamid gel. $t_{\mathrm{g}}$ is the gelation threshold $\left(T=16^{\circ} \mathrm{C}\right.$, $v=110 \mathrm{MHz}$ ). At $t=0$ the attenuation is that of the monomer solution.

1.1 Chemical gelation in $10 \%$ POlyacrylamid GEL (Figs. 1 and 2). - For the same concentration, the gelation has been observed on two samples maintained at $20^{\circ} \mathrm{C}$ and $16^{\circ} \mathrm{C}$ and the gelation starts

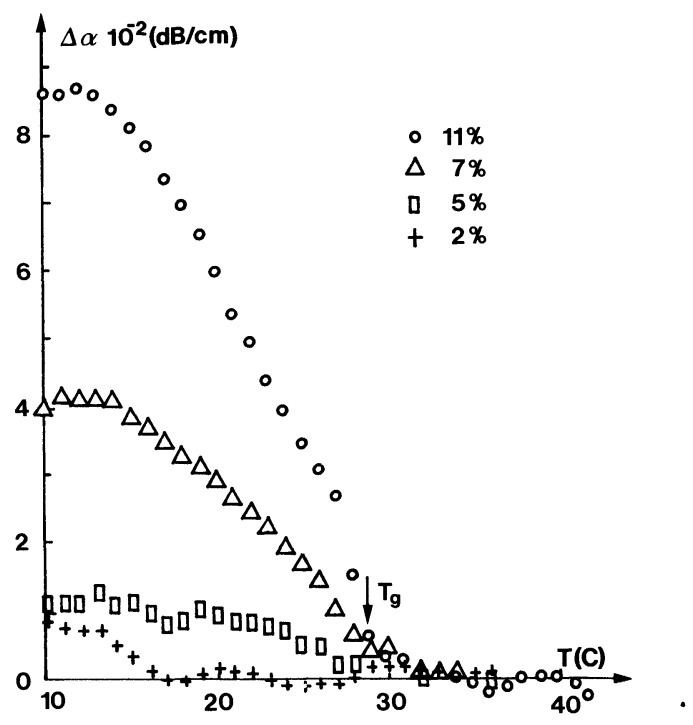

Fig. 3. - Difference of the ultrasonic attenuation between the gelatin and water versus temperature. $T_{\mathrm{g}}$ is the gelation threshold for the $11 \%$ gelatin. $v=790 \mathrm{MHz}$.

$240 \mathrm{~s}$ and $460 \mathrm{~s}$ respectively after the mixing. Figures 1 and 2 are drawings of the variations $\Delta \alpha$ of the attenuation observed during the gelation, at $20^{\circ} \mathrm{C}$ and $16{ }^{\circ} \mathrm{C}$. The attenuation shows a sudden increase, and tends to saturate with the slowing down of the reaction. The maximum attenuation attained after a sufficiently long time $(\simeq 4000 \mathrm{~s})$ is the same for the two different temperatures, when the gel is completely formed.

The variation $\Delta \alpha$ as a function of $t-t_{\mathrm{g}}, t_{\mathrm{g}}$ being the value where the attenuation starts increasing, is linear from 0 to $150 \mathrm{~s}$ and $400 \mathrm{~s}$, at $20^{\circ} \mathrm{C}$ and $16^{\circ} \mathrm{C}$ respectively.

1.2 Physical gelation in Gelatin (Figs. 3 and 4). - Figure 3 shows the difference $\Delta \alpha$ between the

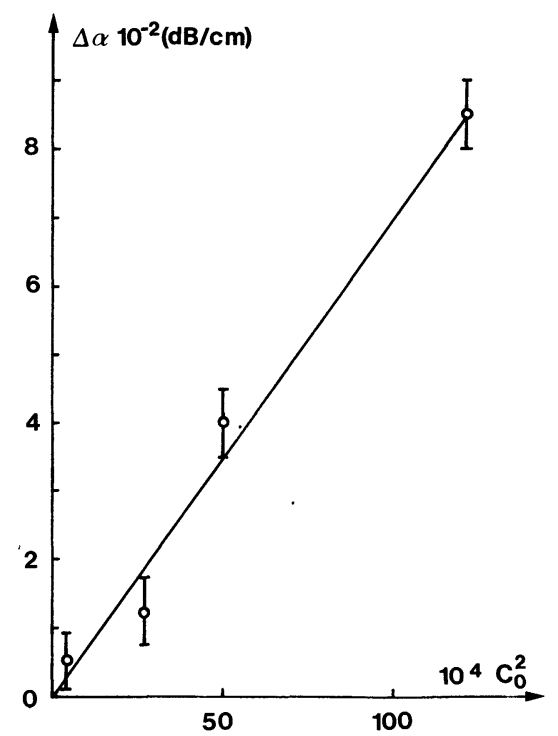

Fig. 4. - Ultrasonic attenuation in the gelatin at $10^{\circ} \mathrm{C}$ versus the squared concentration. 
attenuation observed between $10^{\circ} \mathrm{C}$ and $40^{\circ} \mathrm{C}$, and the water attenuation at the same temperatures. The curves obtained are far from being as demonstrative of the effect as in the case of polyacrylamid gel. However, for the $7 \%$ and $11 \%$ concentrations, the change of slope defining $T_{\mathrm{g}}$ is quite clearly seen. From this point, the variation of $\Delta \alpha$ versus $T_{\mathrm{g}}-T$ is roughly linear.

The attenuation plateau obtained at $10^{\circ} \mathrm{C}$ in the gelatin has been plotted as a function of $c_{0}^{2}$. Figure 4 shows that the attenuation plateau value obtained at $10^{\circ} \mathrm{C}$ is linear as a function of $c_{0}^{2}$. ( $c_{0}$ is the gelatin concentration.)

The ratio $\Delta \alpha_{\text {plateau }} / v^{2}$ ( $v:$ ultrasonic frequency) has the same order of magnitude in the two gelating systems (near 1 and $4 \times 10^{15} \mathrm{~dB} \mathrm{~s}^{2} / \mathrm{cm}$ ).

2. Theory. - In a previous ultrasonic study of the spinodal transition in a polyacrylamid gel [6], a theory has been used which considers a gel as a two-component system [7] : the solvent and the infinite cross-linked network. The coupling between the two component movement is insured by a friction coefficient $f$ between the fluid and the polymer network. This model gives the expression for the attenuation $\alpha$ of a longitudinal acoustic wave of frequency $v$ :

$$
\frac{\alpha}{v^{2}}=\frac{2 \pi^{2}}{\left(\rho_{\mathrm{s}}+c_{0} \rho_{\mathrm{r}}\right) v^{3}}\left(\frac{4}{3} \eta_{\mathrm{s}}+\xi_{\mathrm{s}}+\frac{c_{0}^{2} \rho_{\mathrm{r}} E_{\mathrm{s}}}{\rho_{\mathrm{s}} f}\right)
$$

and the ultrasonic velocity $v$ :

$$
v \simeq \sqrt{\frac{E_{\mathrm{s}}}{\rho_{\mathrm{s}}+c_{0} \rho_{\mathrm{r}}}}
$$

where $\rho_{\mathrm{r}}$ is the density of the network, $\rho_{\mathrm{s}}$ and $E_{\mathrm{s}}$ are the density and the longitudinal elastic constant of the solvent, $\frac{4}{3} \eta_{\mathrm{s}}+\xi_{\mathrm{s}}$ is the solvent viscosity for a longitudinal wave, and $c_{0}$ is the polymer concentration.

This model which has been applied in the case of a completely formed gel can explain the present experiments with slight modifications. We have now to explain why the attenuation is constant in the sol phase and grows when the gel fraction is in formation.

- In the sol phase different mechanisms are possibly efficient to give ultrasonic attenuation and we are going to rule them out successively.

- The divergence of the macroscopic viscosity results from slow motions of the polymers which are not excited in our high frequencies experiments. The effective viscosity is then very close to the solvent viscosity $\left(\frac{4}{3} \eta_{\mathrm{s}}+\xi_{\mathrm{s}}\right)$. (The frequency of entanglements [7] which is the limit between these two regimes ranges from $10^{3}$ to $10^{4} \mathrm{~Hz}$ and decreases towards the transition.)

- The scattering of elastic waves on the clusters and viscous drag effect could occur at our frequencies resulting in an attenuation of the transmitted signal. However the cross-sections depend on the difference between the bulk modulus and density of the solvent and the gel cluster respectively [8] : these differences are negligible as a gel is mainly composed of solvent.

- However when the gel clusters dimensions are of the same order as the wavelength, there is a pressure phase difference between different points of the same cluster and the solution begins to be seen as a solid-like medium with a certain elastic constant. This situation still occurs theoretically in the sol phase but is experimentally sufficiently close to $T_{\mathrm{g}}$ to very well define the transition temperature. (At $100 \mathrm{MHz}$, the wavelength is near $15 \mu$ and the correlation length is estimated to have this value at $5 / 1000 \mathrm{~K}$ of $T_{\mathrm{g}}$ ) In these conditions the ultrasonic attenuation depends on the concentration of the gel fraction in the gel phase. If $c_{0}$ is the monomer concentration and $S_{\infty}$ the gel fraction, the attenuation is then written more satisfactorily :

$$
\frac{\alpha}{v^{2}}=\frac{2 \pi^{2}}{\left(\rho_{\mathrm{s}}+c_{0} \rho_{\mathrm{r}}\right) v^{3}}\left(\frac{4}{3} \eta_{\mathrm{s}}+\xi_{\mathrm{s}}+\frac{c_{0}^{2} S_{\infty}^{2} \rho_{\mathrm{r}} E_{\mathrm{s}}}{\rho_{\mathrm{s}} f}\right) .
$$

The term $\frac{4}{3} \eta_{\mathrm{s}}+\xi_{\mathrm{s}}$ relative to the solvent is not important, composes the background of the signal, and may be subtracted : the remaining term $\Delta \alpha / v^{2}$ is written using the expression of $v$ and the fact that $E_{\mathrm{s}} \gg E_{\mathrm{r}}$ :

$$
\frac{\Delta \alpha}{v^{2}}=\frac{2 \pi^{2}}{v} \frac{\rho_{\mathrm{r}}}{\rho_{\mathrm{s}}} c_{0}^{2} \frac{S_{\infty}^{2}}{f}
$$

The attenuation difference between the gel phase and the sol phase is a measurement of the size of $S_{\infty}^{2} / f$.

Our experimental results can be discussed in the light of this theory.

- The values of the attenuation plateau occurring far from $T_{\mathbf{g}}$ in gelatin reflect only the variations in monomer concentration $c_{0}, S_{\infty}$ being supposed to be 1 in this region where the gel formation is completed. Figure 4 supports this assumption.

- For the polyacrylamid gel, the attenuation plateau values obtained for times long after $t_{\mathrm{g}}$, do not depend on the reaction temperature : the state of the formed gel is the same in the two cases.

- The slope of $\Delta \alpha$ is a linear function of $t-t_{\mathrm{g}}$ (or $T_{\mathrm{g}}-T$ ) in the vicinity of $t_{\mathrm{g}}$ (or $T_{\mathrm{g}}$ ). This experimental result is obvious in the polyacrylamid gel, for the two reaction temperatures, a little less evident in the gelatin case where the signal is not so good.

It is difficult in our experiments to know the relation between the time (or the temperature) and the parameter $p$, the fraction of reacted bonds. We suppose a linear relation between $t-t_{\mathrm{g}}\left(\right.$ or $\left.T_{\mathrm{g}}-T\right)$ 
and $p-p_{\mathrm{c}}$ when $p$ is sufficiently close to $p_{\mathrm{c}}$, the critical threshold and we then give the result :

$$
\frac{S_{\infty}^{2}}{f} \propto\left(p-p_{\mathrm{c}}\right) .
$$

Far from $p_{\mathrm{c}}$ the reaction kinetics are strongly modified by the decreasing of the diffusion possibilities for the different reacting chemical species and this approximation is no longer possible.

A further step can be made with certain assumptions : the friction coefficient $f$ which appears in the attenuation is a measurement of the interaction between the solvent and the polymer in a certain volume of the order of $\lambda^{3}, \lambda$ is the wavelength of the ultrasonic wave. The friction is certainly very sensitive to the distribution of polymer in this volume : for instance, near the gel spinodal transition, when the chains shrink around the tie points and are stretched between, the friction is lowered [6]. Near the threshold, a cluster in the sol phase has already the same geometry as the infinite cluster in the gel phase : in particular, the mean distance between tie points depends on the cross-linking agent concentration among other factors of the chemical reaction. When the infinite cluster is formed at $p_{\mathrm{c}}$, the friction coefficient $f$, which is sensitive to the cell dimensions in the gel, has no reason to vary strongly and can be considered as being a constant.

If these assumptions, which seems to be supported by some experiments [3], are right, our experimental curves would give the critical exponent $\beta$ of the gel fraction, $S_{\infty} \propto\left(p-p_{\mathrm{c}}\right)^{\beta}$ :

$$
\beta=0.5 \text {. }
$$

- U. Brauner [11] carried out a literature search among papers related to gelation and calculated the $\beta$ indices from the published curves : the $\beta$ values are distributed widely between 0.4 and 1 .

- Some numerical calculations have been made on percolation models : the value of $\beta$ depends on the dimensionality of space but does not seem to be function of the type of lattice postulated in these evaluations. However the calculation is done on a very small number of linking points compared to the real conditions. For 3-dimensional space, the result is $0.3 \lesssim \beta \lesssim 0.4[8]$.

3. Conclusion. - The measurement of the ultrasonic attenuation in system undergoing a gelation réaction gives a direct measurement of $S_{\infty}^{2} / f$ monitoring the extent of the gel fraction into the melt. If the friction coefficient is taken as a constant in the gel phase, the critical exponent $\beta$ of the gel fraction $S_{\infty}$ is found to be equal to 0.5 .

The method is sensitive and does not destroy the very fragile structure of the gel in the very close region of $p_{\mathrm{c}}$.

\section{References}

[1] DE Gennes, P. G., J. Physique Lett. 40 (1979) L-197.

[2] Dumas, J., and BaCRI, J.-C., J. Physique Lett. 41 (1980).

[3] BaCri, J.-C. and Dumas, J., J. Physique Lett. 41 (1980).

[4] Adam, M., Delsanti, M., Okasha, R., and Hild, G., $J$. Physique Lett. 40 (1979) L-539.

[5] BaCri, J.-C., Dumas, J. and Levelut, A., J. Physique Lett. 40 (1979) L-231.

[6] BaCri, J.-C. and Rajaonarison, R., J. Physique Lett. 40 (1979) L-5.
[7] DE GeNnes, P. G., Macromolécules 9 (1976) 587 ; 574.

[8] Wada, Y., Hirose, H., Umebayashi, H., OtOMo, M., J. Phys. Soc. Japan 15 (1960) 2324.

[9] Froelich, B., Nö̈L, C. and Monnerie, L., Polymer 20 (1979) 529.

[10] Pugh, D. and Jones, D. A., Polymer 19 (1978) 1008.

[11] Brauner, U., Makromol. Chem. 180 (1979) 251.

[12] Kirpatrick, S., Rev. Mod. Phys. 45 (1973) 45. 